\title{
SHIFTED CONVOLUTION $L$-SERIES VALUES FOR ELLIPTIC CURVES
}

\author{
ASRA ALI AND NITYA MANI
}

\begin{abstract}
Using explicit constructions of the Weierstrass mock modular form and Eisenstein series coefficients, we obtain closed formulas for the generating functions of values of shifted convolution $L$-functions associated to certain elliptic curves. These identities provide a surprising relation between weight 2 newforms and shifted convolution $L$-values when the underlying elliptic curve has modular degree 1 with conductor $N$ such that $\operatorname{genus}\left(X_{0}(N)\right)=1$.
\end{abstract}

\section{INTRODUCTION}

The Modularity Theorem [3] and Eichler-Shimura theory [11, 21] enable weight 2 newforms for $\Gamma_{0}(N)$ with integral coefficients to be uniquely associated to isogeny classes of elliptic curves over $\mathbb{Q}$. Within these results are explicit methods for constructing the 2dimensional complex lattice $\Lambda_{E}$ associated to an elliptic curve $E$, given a weight 2 newform.

Throughout the paper, fix the weight 2 newform associated to an elliptic curve $E / \mathbb{Q}$ as

$$
f_{E}(z)=\sum_{n=1}^{\infty} a_{E}(n) q^{n} ; \quad q=e^{2 \pi i z} ; \quad z \in \mathcal{H}
$$

where $\mathcal{H}$ is the upper half-plane. We denote the complex analytic realization of an elliptic curve as $\mathbb{C} / \Lambda_{E}$, and its modular parametrization as $\phi_{E}: X_{0}(N) \rightarrow \mathbb{C} / \Lambda_{E}$. The modular degree is the degree of the map $\phi_{E}$. In this paper, we only consider elliptic curves $E$ of modular degree 1 with conductor $N$ such that genus $\left(X_{0}(N)\right)=1$, which restricts $N$ to the finite set $\{11,14,15,17,19,21,27,32,36,49\}$.

Our expressions make use of the Weierstrass mock modular form associated to $E$ (a more detailed exposition can be found in Section 2.2). These functions were recently introduced by Alfes, Griffin, Guerzhoy, Ono, and Rolen in [1, 14] and arise from the Weierstrass $\zeta$-function. The Weierstrass $\zeta$-function of an elliptic curve $E$ with complex analytic realization $\mathbb{C} / \Lambda_{E}$ is defined by

$$
\zeta\left(\Lambda_{E} ; w\right)=\frac{1}{w}+\sum_{x \in \Lambda_{E} \backslash\{0\}}\left(\frac{1}{w-x}+\frac{1}{x}+\frac{w}{x^{2}}\right) .
$$

It is related to the classical Weierstrass $\wp$-function by differentiation

$$
\frac{d}{d w} \zeta\left(\Lambda_{E} ; w\right)=-\wp\left(\Lambda_{E} ; w\right)
$$

Date: May 15, 2019. 
We also consider $\mathcal{E}_{f_{E}}(z)$, the Eichler integral of $f_{E}$ :

$$
\mathcal{E}_{f_{E}}(z)=\sum_{n=1}^{\infty} \frac{a_{E}(n)}{n} q^{n}
$$

Note that this is essentially the antiderivative of the newform $f_{E}$ associated to the elliptic curve $E$.

Although the Weierstrass $\wp$-function is doubly periodic with respect to $\Lambda_{E}$, the $\zeta$-function is not. Motivated by this, Eisenstein (see [16]) constructed a modification of $\zeta\left(\Lambda_{E} ; w\right)$, a lattice invariant, non-holomorphic function $\mathfrak{\mathfrak { Z }}_{E}(w)$, defined by

$$
\mathfrak{Z}_{E}(w)=\zeta\left(\Lambda_{E} ; w\right)-S\left(\Lambda_{E}\right) w-\frac{\pi}{\operatorname{vol}\left(\Lambda_{E}\right)} \bar{w},
$$

where

$$
S\left(\Lambda_{E}\right)=\lim _{s \rightarrow 0} \sum_{x \in \Lambda_{E} \backslash\{0\}} \frac{1}{x^{2}|x|^{2 s}} .
$$

This value $S\left(\Lambda_{E}\right)$ is essentially the weight 2 Eisenstein series for the lattice $\Lambda_{E}$. Using this, we define the function $\widehat{\mathfrak{Z}}_{E}(z)$ as the evaluation of $\mathfrak{Z}_{E}(z)$ at the holomorphic Eichler integral $\mathcal{E}_{f_{E}}(z)$,

$$
\widehat{\mathfrak{Z}}_{E}(z)=\mathfrak{Z}_{E}\left(\mathcal{E}_{f_{E}}(z)\right) .
$$

Then $\widehat{\mathfrak{Z}}_{E}(z)$ is a harmonic Maaß form (see Section 2.2 and can be written as a sum of a holomorphic and non-holomorphic part

$$
\widehat{\mathfrak{Z}}_{E}(z)=\widehat{\mathfrak{Z}}_{E}^{+}(z)+\widehat{\mathfrak{Z}}_{E}^{-}(z) .
$$

We refer to the holomorphic function $\widehat{\mathfrak{Z}}_{E}^{+}(z)$ as the Weierstrass mock modular form associated to $E$. When the modular degree is 1 (as in the curves we study) $\widehat{\mathfrak{Z}}_{E}^{+}(z)$ is the holomorphic part of the weight 0 harmonic Maaß form $\widehat{\mathfrak{Z}}_{E}(z)$ (see the work of Alfes, Griffin, Ono and Rolen in [1]), making this terminology appropriate.

The Hasse-Weil $L$-function $L(E, s)=L\left(f_{E}, s\right)$ plays a central role in the arithmetic of $E$. Indeed, the Birch and Swinnerton-Dyer Conjecture asserts that the arithmetic invariants of $E / \mathbb{Q}$ are encoded by the analytic behavior of $L(E, s)$ at $s=1$. We can also consider shifted convolution $L$-functions associated to an elliptic curve $E$. Here, we consider the shifted convolution $L$-functions evaluated at $s=1$ defined by

$$
D_{f_{E}}(h ; s)=\sum_{n=1}^{\infty} a_{E}(n+h) \overline{a_{E}(n)}\left(\frac{1}{(n+h)^{s}}-\frac{1}{n^{s}}\right) .
$$

For convenience, we denote the generating function of these values in $h$-aspect by

$$
\mathbb{L}_{f_{E}}(z)=\sum_{h=1}^{\infty} D_{f_{E}}(h ; 1) q^{h} .
$$

Shifted convolution $L$-functions were defined by Hoffstein, Hulse, and Reznikov 13. They are generalizations of the classical Rankin-Selberg convolutions [17], 20] which were used to bound the growth of the Fourier coefficients of cusp forms. Properties of these shifted 
convolution Dirichlet series have been investigated recently; it was first shown that these shifted convolution values are essentially coefficients of mixed mock modular forms (see [15]) and later the $p$-adic properties of these series (see [4]) and their asymptotic behavior (see [2]) were investigated.

Initially motivated by a desire to compute these $L$-values and understand the explicit construction of the Weierstrass mock modular form (in terms of other known elliptic invariants), we offer a closed formula for these generating functions $\mathbb{L}_{f_{E}}(z)$. We provide such formulas for newforms associated to elliptic curves $E$ of modular degree 1 and conductor $N$ such that genus $\left(X_{0}(N)\right)=1$.

These results contribute to the existing theory of shifted convolution $L$-series for the subset of curves we study. To make this more precise, let $F_{N, 2}^{\rho(i)}$ be the (quasimodular) Eisenstein series for $\Gamma_{0}(N)$ nonvanishing and normalized to be 1 only at the cusp $\rho(i)$ and vanishing at all other cusps (as in Section 2.5). Throughout this paper, for any 1-periodic function $f$, let $f[h]$ denote the coefficent of $q^{h}$ in the Fourier expansion of $f$, and recall that $f_{E}=\sum_{n=1}^{\infty} a_{E}(n) q^{n}$ denotes the weight 2 newform associated to the elliptic curve $E$.

For the first result, we restrict to the case where $N$ is squarefree and thus $N$ lies in the set $\{11,14,15,17,19,21\}$.

Theorem 1.1. Assume the notation and hypotheses above. Then, we have that

where

$$
\mathbb{L}_{f_{E}}(z)=\frac{\operatorname{vol}\left(\Lambda_{E}\right)}{\pi}\left(\left(f_{E}(z) \cdot \widehat{\mathfrak{Z}}_{E}^{+}(z)\right)-\alpha f_{E}(z)-F_{N, 2}^{\infty}(z)\right)
$$

$$
\alpha=\left(f_{E} \cdot \widehat{\mathfrak{Z}}_{E}^{+}\right)[1]-\frac{\pi}{\operatorname{vol} \Lambda_{E}} D_{f_{E}}(1 ; 1)-F_{N, 2}^{\infty}[1] .
$$

A special case occurs when we drop the condition that $N$ is squarefree and instead suppose that $E$ has complex multiplication, restricting $N$ to be in the set $\{27,32,36\}$ (the result does still hold for $N=49$ numerically, but is excluded from the this set due to a technical limitation). In this case, computational evidence proves that $\alpha=0$, yielding the following stronger result.

Theorem 1.2. Let $E$ be an elliptic curve as above with complex multiplication and conductor $N \neq 49$. With $\mathbb{L}_{f_{E}}(z)$ defined as above, we have

$$
\mathbb{L}_{f_{E}}(z)=\frac{\operatorname{vol}\left(\Lambda_{E}\right)}{\pi}\left(\left(f_{E}(z) \cdot \widehat{\mathfrak{Z}}_{E}^{+}\right)(z)-F_{N, 2}^{\infty}(z)\right)
$$

These identities use the characterization of holomorphic projection given by Mertens and Ono in 15 to explicitly relate the Fourier coefficients of weight 2 newforms to elliptic curve invariants and to understand the behavior of the Weierstrass mock modular form. These identities can also be used to compute the shifted convolution $L$-values to arbitrary precision.

In the following section, we provide some preliminaries on harmonic Maaßforms, mock modular forms, and characterize the Weierstrass mock modular form. We also introduce Maaß-Poincaré series and holomorphic projection which are connected to the shifted convolution $L$-series we study. This enables us in Section 3 to compute the generating function for the shifted convolution $L$-series in the desired cases, as well as prove vanishing properties 
of the shifted convolution Dirichlet series and holomorphic projection associated to a subset of elliptic curves. Finally, we give examples of our explicit results in the case of conductors $N=11$ and $N=27$.

\section{Preliminaries}

2.1. Harmonic Maaß forms and mock modular forms. We begin with a review of harmonic Maaß forms. These real-analytic modular forms were first introduced by Bruinier and Funke in [6]. Among many other important roles these forms play in number theory, work by Zwegers (see [25]) shows that Ramanujan's mock $\theta$-functions arise as holomorphic parts of harmonic Maaß forms. These forms are also intimately connected to our study of the holomorphic projections associated to certain elliptic curves.

Let $\mathcal{H}=\{x+i y: x, y \in \mathbb{R}, y>0\}$ denote the upper half-plane. Consider $z=x+i y \in \mathcal{H}$ and as noted in the introduction, let $q=e^{2 \pi i z}$. For any $\gamma=\left[\begin{array}{ll}a & b \\ c & d\end{array}\right] \in \mathrm{SL}_{2}(\mathbb{Z})$ we abbreviate the action of $\gamma$ on a function $f$ by the Petersson slash operator of weight $k$,

$$
\left(\left.f\right|_{k} \gamma\right)(z)=(c z+d)^{-k} f\left(\frac{a z+b}{c z+d}\right)
$$

Consider the congruence subgroup of level $N$, defined as follows:

$$
\Gamma_{0}(N)=\left\{\left[\begin{array}{ll}
a & b \\
c & d
\end{array}\right] \in \mathrm{SL}_{2}(\mathbb{Z}) \mid c \equiv 0 \bmod N\right\} .
$$

Definition 2.1. ([6]) A weak harmonic Maaß form of weight $k \in \mathbb{Z}$ on $\Gamma_{0}(N)$ is a smooth function $f$ on $\mathcal{H}$ that satisfies the following three conditions:

(1) $\left(\left.f\right|_{k} \gamma\right)(z)=f(z)$ for all $\gamma \in \Gamma_{0}(N)$ (i.e. $f$ transforms like a modular form on $\Gamma_{0}(N)$ ),

(2) $\Delta_{k} f(z) \equiv 0$, where if $z=x+i y, \Delta_{k}=-y^{2}\left(\frac{\partial^{2}}{\partial x^{2}}+\frac{\partial^{2}}{\partial y^{2}}\right)+i k y\left(\frac{\partial}{\partial x}+i \frac{\partial}{\partial y}\right)$

(3) $f(z)$ has poles at most at the cusps of $\Gamma_{0}(N)$ (i.e. there exists some polynomial $P(z)$, such that $f(z)-P\left(q^{-1}\right)=O\left(e^{-\alpha y}\right)$ for $\alpha>0$ as $y \rightarrow \infty$ and an analogous condition holds for the other cusps of $\left.\Gamma_{0}(N)\right)$.

Note that hereafter, we will simply term such smooth $f$ as weight $k$ harmonic Maaß forms for $\Gamma_{0}(N)$. Following immediately from the definition above, we see that a harmonic Maaß form admits a Fourier expansion of the following form:

Lemma 2.2 ( [6]). A weight $k$ harmonic Maaß form $f(z)$ admits a Fourier expansion of the form,

$$
\begin{gathered}
f(z)=f^{+}(z)+f^{-}(z) ; \\
f^{+}(z)=\sum_{n \gg-\infty} c_{f}^{+}(n) q^{n} ; \quad f^{-}(z)=\sum_{n=1}^{\infty} c_{f}^{-}(n) q^{n} \Gamma(k-1,4 \pi n y) .
\end{gathered}
$$

Here, $\Gamma(\alpha, \beta)=\int_{\beta}^{\infty} e^{-t} t^{\alpha-1} d t$ denotes the incomplete Gamma function, and $f^{+}(z)$ and $f^{-}(z)$ are the holomorphic and non-holomorphic parts of $f(z)$ respectively.

This construction also gives rise to a characterization of mock modular forms in terms of harmonic Maaß forms: 
Definition 2.3. Consider $f(z)=f^{+}(z)+f^{-}(z)$ as defined above. If $f^{-}(z) \neq 0$, then $f^{+}(z)$ is called a mock modular form.

We define a differential operator (as in [16]) to help characterize $f^{-}(z)$ when it is nontrivial. Denote by $H_{k}(\Gamma)$ the space of weight $k$ harmonic Maaß forms for some congruence subgroup $\Gamma \leq \mathrm{SL}_{2}(\mathbb{Z})$.

Proposition 2.4 ( $[6])$. Define a differential operator $\xi_{2-k}: H_{2-k}(\Gamma) \rightarrow S_{k}(\Gamma)$ where $\Gamma \leq \mathrm{SL}_{2}(\mathbb{Z})$ as

$$
\xi_{2-k}(f(z))=2 i y^{k} \frac{\overline{\partial f}}{\partial \bar{z}} .
$$

Then $\xi_{2-k}$ is a well-defined, surjective, antilinear map with kernel $M_{2-k}^{!}(\Gamma)$, the space of weakly holomorphic weight $2-k$ modular forms for $\Gamma$. Further,

$$
\xi_{2-k} f(z)=-(4 \pi)^{k-1} \sum_{n=1}^{\infty} c_{f}^{-}(n) q^{n} .
$$

We call the function defined by $\sum_{n=1}^{\infty} c_{f}^{-}(n) q^{n}$ the shadow of $f(z)$ or $f^{+}(z)$. When the shadow is non-trivial, we obtain some conditions on the cusp behavior of $f(z)$.

Proposition 2.5. (Lemma 2.3 of [5]) If $f(z) \in H_{2-k}\left(\Gamma_{0}(N)\right)$ has the property that $\xi_{2-k}(f) \neq$ 0 , then the principal part of $f(z)$ is nonconstant for at least one cusp.

The kernel of the surjective $\xi_{2-k}$ operator defined above is infinite dimensional. Selecting a suitable class of harmonic Maaß forms to serve as preimages under this $\xi$ operator depends intimately on the following notion:

Definition 2.6. A harmonic Maaß form $F(z) \in H_{2-k}\left(\Gamma_{0}(N)\right)$ is $\operatorname{good}$ for $f(z) \in S_{k}\left(\Gamma_{0}(N)\right)$ if it satisfies the following 3 conditions:

(1) The principal part of $F(z)$ at the cusp $\infty$ is in $\mathbb{F}_{f}\left[q^{-1}\right]$, where $\mathbb{F}_{f}$ is the field obtained by adjoining the Fourier coefficients of $f$ to $\mathbb{Q}$.

(2) The principal part of $F(z)$ at all other inequivalent cusps of $\Gamma_{0}(N)$ is constant.

(3) $\xi_{2-k}(F(z))=\|f(z)\|^{-2} f(z)$, where $\xi_{2-k}$ is the differential operator defined in Proposition 2.4

2.2. Weierstrass mock modular forms. We begin with the construction of the Weierstrass mock modular form associated with an elliptic curve $E$ of conductor $N$. This will prove very useful to our analysis of shifted convolution $L$-series values. The Weierstrass mock modular form was introduced in [14] as a mechanism by which to understand properties of elliptic curves and their associated newforms through the language of harmonic Maaß forms. The Weierstrass mock modular form has been one of the primary objects of study by many recently, as in 1,9 .

The theory of elliptic curves gives rise to a notable example of a weight 0 harmonic Maaß form. Recall that $E \simeq \mathbb{C} / \Lambda_{E}$ where $\Lambda_{E}$ is a 2-dimensional lattice in $\mathbb{C}$. Recall the Weierstrass $\zeta$-function defined in (1.2). Although it is not elliptic, its derivative is negative the Weierstrass $\wp$-function. This relation gives the Laurent expansion of $\zeta$ : 
Proposition 2.7. [10] The Laurent expansion of $\zeta$ is

$$
\zeta\left(\Lambda_{E} ; z\right)=\frac{1}{z}-\sum_{k=1}^{\infty} G_{k+2}\left(\Lambda_{E}\right) z^{2 k+1}
$$

where $G_{k}\left(\Lambda_{E}\right)$ is the Eisenstein series of weight $k$ associated to a lattice $\Lambda_{E}$.

Again, recall the construction of the Weierstrass mock modular form given in (1.4):

$$
\widehat{\mathfrak{Z}}_{E}^{+}(z)=\mathfrak{Z}_{E}^{+}\left(\mathcal{E}_{f_{E}}(z)\right)=\zeta\left(\Lambda_{E} ; \mathcal{E}_{f_{E}}(z)\right)-S\left(\Lambda_{E}\right) \mathcal{E}_{f_{E}}(z) .
$$

The following theorem outlines some important properties about the Weierstrass mock modular form.

Theorem 2.8. ([1]) Assume the notation and hypotheses above. Then

(1) The holomorphic part $\widehat{\mathfrak{Z}}_{E}^{+}(z)=\zeta\left(\Lambda_{E} ; \mathcal{E}_{f_{E}}(z)\right)-S\left(\Lambda_{E}\right) \mathcal{E}_{f_{E}}(z)$ has poles exactly when $\mathcal{E}_{f_{E}}(z)$ is a lattice point.

(2) If $\widehat{\mathfrak{Z}}_{E}^{+}(z)$ has poles in the upper half plane, there is a canonical meromorphic modular function $M_{E}(z)$ such that $\widehat{\mathfrak{Z}}_{E}^{+}(z)-M_{E}(z)$ is holomorphic on $\mathcal{H}$.

(3) $\widehat{\mathfrak{Z}}_{E}(z)-M_{E}(z)$ is a harmonic Maaß form of weight 0 on $\Gamma_{0}(N)$ and $\xi_{0}\left(\widehat{\mathfrak{Z}}_{E}^{+}(z)-\right.$ $\left.M_{E}(z)\right)=-(4 \pi) \cdot f_{E}(z)$. In particular, $\widehat{\mathfrak{Z}}_{E}^{+}(z)-M_{E}(z)$ is a weight 0 mock modular form.

In particular, $\widehat{\mathfrak{Z}}_{E}^{+}(z)-M_{E}(z)$ is called the Weierstrass mock modular form of $E$.

In particular, we will be interested in the case where $\widehat{\mathfrak{Z}}_{E}^{+}(z)$ itself is a mock modular form of weight 0 . This arises when the canonical $M_{E}(z)$ is identically zero. The following proposition gives sufficient conditions for this to occur.

Lemma 2.9. Let $E$ be an elliptic curve of conductor $N$ with modular parametrization $\phi_{E}: X_{0}(N) \rightarrow E$. If the modular degree $\operatorname{deg}\left(\phi_{E}\right)$ is 1 , then $\widehat{\mathfrak{Z}}_{E}^{+}(z)$ does not have poles in the upper half-plane.

Proof. Recall the mock modular form $\widehat{\mathfrak{Z}}_{E}^{+}(z)$ is given by Equation (1.4) with Laurent expansion as in Proposition 2.7

$$
\begin{aligned}
\widehat{\mathfrak{Z}}_{E}^{+}(z) & =\mathfrak{Z}_{E}\left(\mathcal{E}_{f_{E}}(z)\right) \\
& =\frac{1}{\mathcal{E}_{f_{E}}(z)}-\sum_{k=1}^{\infty} G_{2 k+2}\left(\Lambda_{E}\right) \mathcal{E}_{f_{E}}(z)^{2 k+1}-S\left(\Lambda_{E}\right) \mathcal{E}_{f_{E}}(z) .
\end{aligned}
$$

The Eichler integral $\mathcal{E}_{f_{E}}(z)=\sum_{n=1}^{\infty} \frac{a_{E}(n)}{n} q^{n}$ is holomorphic on the upper half-plane, so it suffices to show that $\mathcal{E}_{f_{E}}(z)$ does not vanish for any $z \in \mathcal{H}$. The modular parametrization $\phi_{E}: X_{0}(N) \rightarrow \mathbb{C} / \Lambda_{E}$ is induced from the map $\phi_{1}: \mathcal{H} \rightarrow \mathbb{C}$. Here $\phi_{1}$ is the map given by

$$
\begin{aligned}
\phi_{1}(z) & =-2 \pi i \int_{z}^{i \infty} f_{E}(\tau) d \tau \\
& =\sum_{n=1}^{\infty} \frac{a_{E}(n)}{n} q^{n}=\mathcal{E}_{f}(z) .
\end{aligned}
$$


If the modular degree $\operatorname{deg}\left(\phi_{E}\right)$ is 1 (requiring that the genus of $X_{0}(N)$ is $1, \operatorname{since} \operatorname{deg}\left(\phi_{E}\right) \geq$ genus $\left(X_{0}(N)\right)$, then the map $\phi_{E}$ is an isomorphism. Thus, $\mathcal{E}_{f_{E}}(z)$ does not vanish for any $z \in \mathcal{H}$ since it vanishes at the cusp $\infty$.

Moreover, $\widehat{\mathfrak{Z}}_{E}^{+}(z)$ has rational Fourier coefficients if $E$ has complex multiplication (see Theorem 1.3 in [7]).

Example. Consider the strong Weil curve of conductor 27 given by the Weierstrass equation $E_{27}: y^{2}+y=x^{3}-7$ (Cremona label 27a1). The weight 2 modular form associated with $E_{27}$ is given by

$$
f_{E_{27}}=q-2 q^{4}-q^{7}+5 q^{13}+4 q^{16}-7 q^{19}+O\left(q^{20}\right) .
$$

Using the Fourier expansion of the Weierstrass $\zeta$-function, the weight 0 mock modular form associated to $\widehat{\mathfrak{Z}}_{E_{27}}^{+}(z)$ is given by

$$
\widehat{\mathfrak{Z}}_{E_{27}}^{+}(z)=q^{-1}+\frac{1}{2} q^{2}+\frac{1}{5} q^{5}+\frac{3}{4} q^{8}-\frac{6}{11} q^{11}-\frac{1}{2} q^{14}+O\left(q^{17}\right) .
$$

2.3. Poincaré series. The modular parametrization of an elliptic curve $E$ is given by a map $\phi_{E}: X_{0}(N) \rightarrow E$ where $X_{0}(N)$ is the compactification of the curve $\Gamma_{0}(N) \backslash \mathcal{H}$. Let $f_{E}$ be the weight 2 newform associated to this parametrization. The Petersson norm of $f_{E}$ is then

$$
\left\|f_{E}\right\|^{2}=\left\langle f_{E}, f_{E}\right\rangle=\int_{z \in \Gamma_{0}(N) \backslash \mathcal{H}}\left|f_{E}(z)\right|^{2} d x \wedge d y .
$$

Using Petersson norms, we can relate the degree of $\phi_{E}$ with the area of the fundamental parallelogram of the period lattice $\Lambda_{E}$, which is the volume of the elliptic curve, $\operatorname{vol}\left(\Lambda_{E}\right)$.

Proposition $2.10([24])$. The volume $\operatorname{vol}\left(\Lambda_{E}\right)$ of an elliptic curve $E$ is

$$
\operatorname{vol}\left(\Lambda_{E}\right)=\frac{4 \pi^{2}\left\|f_{E}\right\|^{2}}{\operatorname{deg}\left(\phi_{E}\right)}
$$

The Petersson inner product can also be used to extract Fourier coefficients of cusp forms through Poincaré series.

A generic index $m$ Poincaré series is given by

$$
\mathbb{P}\left(m, k, \phi_{m}, N ; z\right)=\sum_{\gamma \in \Gamma_{\infty} \backslash \Gamma_{0}(N)}\left(\left.\phi_{m}^{*}\right|_{k} \gamma\right)(z),
$$

where $\Gamma_{\infty}=\left\{\left[\begin{array}{ll}1 & n \\ 0 & 1\end{array}\right]: n \in \mathbb{Z}\right\}$ and $\phi_{m}^{*}(z)=\phi_{m}(y) e^{2 \pi i m x}$ for a function $\phi_{m}: \mathbb{R}_{>0} \rightarrow \mathbb{C}$ which satisfies $\phi_{m}(y)=O\left(y^{\alpha}\right)$ as $y \rightarrow 0$ for some $\alpha \in \mathbb{R}$.

Using this, the classical index $m$ Poincaré series $P(m, k, N ; z)$ and the Maaß-Poincaré series $Q(-m, k, N ; z)$ are defined as

$$
\begin{aligned}
P(m, k, N ; z) & =\mathbb{P}\left(m, k, e^{-m y}, N ; z\right), \\
Q(-m, k, N ; z) & =\frac{1}{(k-1) !} \mathbb{P}\left(-m, 2-k, N, \mathcal{M}_{1-\frac{k}{2}}(-4 \pi m y) ; z\right),
\end{aligned}
$$

where $\mathcal{M}_{s}(y)$ is defined in terms of the $M$-Whittaker function

$$
\mathcal{M}_{s}(y)=|y|^{-\frac{k}{2}} M_{\frac{k}{2} \operatorname{sgn}(y), s-\frac{1}{2}}(|y|)
$$


defined in 23.

We can characterize a set of Maaß-Poincaré series in terms of the above functions, with a Fourier expansion given using Bessel functions and the Kloosterman sum $K(m, n ; c)$ we recall below:

$$
K(m, n ; c)=\sum_{d \bmod c ;(c, d)=1} e^{2 \pi i \frac{m \bar{d}+n d}{c}},
$$

where $\bar{d}$ is the multiplicative inverse of $d$ modulo $c$. We first recall the Fourier expansion of the classical Poincaré series:

Proposition 2.11 (Theorem $8.3[16]$ ). Consider the weight $k$ Poincaré series of index $m$ and level $N, P(m, k, N ; z)$. Then this Poincaré series has a Fourier expansion as $P(m, k, N ; z)=q^{m}+\sum_{n=1}^{\infty} b_{P}(m, k, N ; n) q^{n}$ where $b_{P}(m, k, N ; n)$ can be defined as follows $($ when $k \equiv 0(\bmod 2))$ :

$$
b_{P}(m, k, N ; n)=\left(\frac{n}{m}\right)^{(k-1) / 2}\left(\delta_{m, n}+2 \pi i^{-k} \sum_{c>0 ; N \mid c} J_{k-1}\left(\frac{2 \pi \sqrt{m n}}{c}\right) \frac{K(m, n ; c)}{c}\right) .
$$

The Fourier expansion and behavior of the Poincaré series at the cusps of $\Gamma_{0}(N)$ is explained by the following characterization of the Maaß-Poincaré series:

Proposition 2.12. (6.2 in [7], 3.3 in [18]) If $k \in 2 \mathbb{N}$, and $m, N \geq 1$, then $Q(-m, k, N ; z) \in$ $H_{2-k}\left(\Gamma_{0}(N)\right)$, and has a Fourier expansion of the form

$$
Q(-m, k, N ; z)=Q^{+}(-m, k, N ; z)+Q^{-}(-m, k, N ; z),
$$

where

$$
Q^{+}(-m, k, N ; z)=q^{-m}+\sum_{n=0}^{\infty} b_{Q}(-m, k, N ; n) q^{n}
$$

and for integers $n \geq 0$ we have

$$
\begin{aligned}
& b_{Q}(-m, k, N ; n)=-2 \pi(-1)^{k / 2} \cdot \sum_{\substack{c>0 \\
c \equiv 0}}\left(\frac{m}{n}\right)^{\frac{k-1}{2}} \frac{K(-m, n, c)}{c} \cdot I_{k-1}\left(\frac{4 \pi \sqrt{|m n|}}{c}\right) \\
& b_{Q}(-m, k, N ; 0)=-\frac{2^{k} \pi^{k}(-1)^{\frac{k}{2}} m^{k-1}}{(k-1) !} \cdot \sum_{\substack{c>0 \\
c(\bmod N)}} \frac{K(-m, 0, c)}{c^{k}} .
\end{aligned}
$$

Additionally, the principal part at all other cusps is zero.

The classical Poincaré series $P(m, k, N ; z)$ and the Maaß-Poincaré series are also related by the differential operator $\xi_{2-k}$ in the following proposition.

Proposition 2.13. (2.6 in [15]) If $k \geq 2$ is even and $m, N \geq 1$, then

$$
\xi_{2-k}(Q(-m, k, N ; z))=(4 \pi)^{k-1} m^{k-1}(k-1) \cdot P(m, k, N ; z) \in S_{k}\left(\Gamma_{0}(N)\right) \text {. }
$$


2.4. Holomorphic projection and shifted convolution Dirichlet series. The study of holomorphic projection is motivated by a desire to understand smooth functions $f$ which transform like modular forms and have "moderate growth" at cusps. A function $f$ with such properties defines a linear functional on the space of cusp forms via the Petersson inner product, and thus we can associate to to $f$ the cusp form defining the same linear functional as $f$. This cusp form is essentially the holomorphic projection of $f$. The holomorphic projection was first introduced in 22] by Sturm and further developed in the work of Gross and Zagier in 12. We will use it here to give a closed-form algebraic characterization of the shifted convolution $L$-series values for some modular forms associated to elliptic curves. Our approach follows previous work as in [4, 15].

We define the holomorphic projection for continuous functions $f: \mathcal{H} \rightarrow \mathbb{C}$, where $f(z)=$ $\sum_{n \in \mathbb{Z}} a(n, y) q^{n}$ that transform like a modular form of weight $k \geq 2$ for $\Gamma_{0}(N)$ and have moderate growth at the cusps. We can make this idea more precise. Suppose the cusps of $\Gamma_{0}(N)$ are $\rho(i)$ (where $\rho(1)$ is chosen to be the infinite cusp), and consider $\sigma_{i} \in \mathrm{SL}_{2}(\mathbb{Z})$ so that $\sigma_{i} \infty=\rho(i)$. Then $f$ has moderate growth at the cusps if for $n>0$

$$
a(n, y)=O\left(y^{2-k}\right), \quad y \rightarrow 0,
$$

and

$$
\left.f\right|_{k} \sigma_{i}=c_{0}^{(i)}+O\left(\frac{1}{d(y)}\right), \quad y \rightarrow \infty,
$$

where $c_{0}^{(i)}$ is the value of the constant Fourier coefficient when $f$ is evaluated at the cusp denoted $\rho(i)$ and $d(y)$ is some polynomial in $y$.

Definition $2.14([22])$. Consider a continuous function $f(z)=\sum_{n \in \mathbb{Z}} a(n, y) q^{n}$ as above (where $z=x+i y$ ) that transforms like a modular form of weight $k \geq 2$ for $\Gamma_{0}(N)$ and has moderate growth at the cusps. Then, the holomorphic projection of $f(z)$, denoted $\pi_{\text {hol }}(f)(z)$ is constructed as follows:

$$
\begin{gathered}
\pi_{\mathrm{hol}}(f)(z)=c_{0}^{(1)}+\sum_{n=1}^{\infty} c(n) q^{n} \\
c(n)=\frac{(4 \pi n)^{k-1}}{(k-2) !} \int_{0}^{\infty} a(n, y) e^{-4 \pi n y} y^{k-2} d y .
\end{gathered}
$$

The holomorphic projection satisfies several natural properties that arise from its construction:

Proposition $2.15([12)$. Consider $f$ as defined above. Then the holomorphic projection of $f$ satisfies the following three properties.

(1) If $f$ is a holomorphic modular form, $\pi_{\text {hol }}(f)=f$

(2) If $k>2, \pi_{h o l}(f) \in M_{k}\left(\Gamma_{0}(N)\right)$, the space of weight $k$ modular forms for $\Gamma_{0}(N)$. If $k=2, \pi_{h o l}(f) \in M_{2}\left(\Gamma_{0}(N)\right) \oplus \mathbb{C} E_{2}=\widetilde{M}_{2}\left(\Gamma_{0}(N)\right)$, the space of weight 2 quasimodular forms for $\Gamma_{0}(N)$.

(3) $\langle g, f\rangle=\left\langle g, \pi_{h o l}(f)\right\rangle$ for any $g \in S_{k}\left(\Gamma_{0}(N)\right)$.

We can understand the holomorphic projection more explicitly in specific cases, such as the following product of a harmonic Maaß form and a cusp form. 
Proposition 2.16 ([15]). Let $M_{f_{1}}$ be the weight $2-k$ harmonic Maaß form whose shadow is $f_{1} \in S_{k}\left(\Gamma_{0}(N)\right)$ where $f_{1}(z)=\sum_{n=1}^{\infty} a_{1}(n) q^{n}$, so $\xi_{2-k} M_{f_{1}}=-(4 \pi)^{k-1} f_{1}$. Consider also the weight $k$ cusp form for $\Gamma_{0}(N), f_{2}(z)=\sum_{n=1}^{\infty} a_{2}(n) q^{n}$. Suppose that $M_{f_{1}}^{+} \cdot f_{2}(z)$ has moderate growth at all cusps. Then,

$$
\begin{aligned}
\pi_{h o l}\left(M_{f_{1}} \cdot f_{2}\right)(z) & =M_{f_{1}}^{+}(z) \cdot f_{2}(z) \\
& -(k-2) ! \sum_{h=1}^{\infty}\left[\sum_{n=1}^{\infty} a_{2}(n+h) \overline{a_{1}(n)}\left(\frac{1}{(n+h)^{k-1}}-\frac{1}{n^{k-1}}\right)\right] q^{h} .
\end{aligned}
$$

Now consider a strong Weil curve $E$ with associated weight 2 newform $f_{E}$ for $\Gamma_{0}(N)$, where the genus of the modular curve $X_{0}(N)$ is 1 , and let $\widehat{\mathfrak{Z}}_{E}$ be defined as in (1.6). Then, we can compute the holomorphic projection of $f_{E} \cdot \widehat{\mathfrak{Z}}_{E}$ as follows:

Corollary 2.17. Let $E$ be a strong Weil curve with associated weight 2 newform $f_{E}$ for $\Gamma_{0}(N)$, where genus $\left(X_{0}(N)\right)=1$ and $\operatorname{deg}\left(\phi_{E}\right)=1$, and let $\widehat{\mathfrak{Z}}_{E}$ be defined as in (1.6). Then, we have the following:

$$
\pi_{h o l}\left(f_{E} \cdot \widehat{\mathfrak{Z}}_{E}\right)(z)=\frac{\operatorname{vol}\left(\Lambda_{E}\right)}{\pi} f_{E}(z) \widehat{\mathfrak{Z}}_{E}^{+}(z)-\sum_{h=1}^{\infty} D_{f_{E}}(h ; 1) q^{h} .
$$

Proof. Since $\operatorname{dim}\left(S_{2}\left(\Gamma_{0}(N)\right)\right)=\operatorname{genus}\left(X_{0}(N)\right)=1$, the modular form $f_{E}$ is a scalar multiple of the Poincaré series $f_{E}=\frac{1}{\beta} P(1,2, N ; z)$, where $P(m, k, N ; z)$ is the Poincaré series described in Proposition 2.11. Then the Petersson coefficient formula (see 15) and (1.5) yields $\beta=\frac{\pi}{\operatorname{vol}\left(\Lambda_{E}\right)}$. Following the computation in Corollary 1.2 of [15], we obtain the holomorphic projection in terms of the Poincaré series

$$
\pi_{\text {hol }}\left(f_{E} \cdot \widehat{\mathfrak{Z}}_{E}\right)(z)=P(1,2, N ; z) \widehat{\mathfrak{Z}}_{E}^{+}(z)-\mathbb{L}_{f_{E}}(z) .
$$

We can apply Proposition 2.16 with $P(1,2, N ; z)=\frac{1}{\beta} f_{E}$, which yields the Lemma.

Remark. For the remainder of the paper, we will define a new function $\widehat{\pi}_{\text {hol }}$, a scalar multiple of the holomorphic projection by the constant $\frac{\pi}{\operatorname{vol}\left(\Lambda_{E}\right)}$ for ease of algebraic computation and numerical characterization. Thus, we will say

$$
\widehat{\pi}_{\mathrm{hol}}\left(f_{E} \cdot \widehat{\mathfrak{Z}}_{E}\right)(z)=f_{E}(z) \widehat{\mathfrak{Z}}_{E}^{+}(z)-\frac{\pi}{\operatorname{vol}\left(\Lambda_{E}\right)} \mathbb{L}_{f_{E}}(z)
$$

2.5. Eisenstein series. In order to understand $\widehat{\pi}_{\text {hol }}\left(f_{E} \cdot \widehat{\mathfrak{Z}}_{E}\right)$, we define a basis for the space of weight 2 quasimodular forms for $\Gamma_{0}(N)$, the space $\mathcal{E}_{2}\left(\Gamma_{0}(N)\right) \oplus \mathbb{C} E_{2}$, denoted $\widetilde{\mathcal{E}}_{2}\left(\Gamma_{0}(N)\right)$. To do this, we follow the construction given in $\S 2$ of Chapter VII in [19] and arrive at a set of forms $F_{N, 2}^{-a_{2} / a_{1}}$ described below:

Definition 2.18. Define the Eisenstein series $G_{N, k}^{-a_{2} / a_{1}}: \mathcal{H} \rightarrow \widehat{\mathbb{C}}$ for $\Gamma(N), k>2$, as

$$
G_{N, k}^{-a_{2} / a_{1}}(\tau)=\sum_{m_{1} \equiv a_{1}(N), m_{2} \equiv a_{2}(N)}^{\prime}\left(m_{1} \tau+m_{2}\right)^{-k}
$$


where the sum is taken over nonzero integer pairs $\left(m_{1}, m_{2}\right)$ satisfying the congruence conditions described above, where $a=\left(a_{1}, a_{2}\right) \in \mathbb{P}^{1}(\mathbb{Q})$ ranges over the $\Gamma(N)$ inequivalent cusps. Let

$$
\phi_{N, k}^{-a_{2} / a_{1}}(\tau, s)=\sum_{m_{1} \equiv a_{1}(N), m_{2} \equiv a_{2}(N)}^{\prime}\left(m_{1} \tau+m_{2}\right)^{-k}\left|m_{1} \tau+m_{2}\right|^{-s}
$$

with analytic continuation to the $s$-plane as a meromorphic function $\phi_{N, k}^{-a_{2} / a_{1}}$ (that is holomorphic at $s=0)$. Then for $k=2$, define

$$
G_{N, 2}^{-a_{2} / a_{1}}(\tau)=\phi_{N, 2}^{-a_{2} / a_{1}}(\tau, 0)
$$

where $\left(a_{1}, a_{2}\right), N$ are as above.

We can also associate an Fourier expansion to each Eisenstein series $G_{N, k}^{-a_{2} / a_{1}}$ and note that each of these defines a modular form.

Proposition 2.19 (Chapter VII 19$]$ ). For all $a=\left(a_{1}, a_{2}\right), N \geq 2, k>2 G_{N, k}^{-a_{2} / a_{1}}$ is a (holomorphic) modular form weight $k$ for $\Gamma(N)$ with a Fourier expansion given as follows, where $\zeta_{N}=e^{2 \pi i / N}$ and $\delta\left(a_{1} / N\right)=1$ if $a_{1} \equiv 0(\bmod N)$ else is 0 :

$$
\begin{gathered}
G_{N, k}^{-a_{2} / a_{1}}(\tau)=\delta\left(\frac{a_{1}}{N}\right) \sum_{m_{2} \equiv a_{2}(N)}^{\prime} m_{2}^{-k}+\sum_{n>0} \alpha_{n}(N, k, a) \exp (2 \pi i \tau n / N) \\
\alpha_{n}=\frac{(-2 \pi i)^{k}}{N^{k}(k-1) !} \sum_{m \mid n, \frac{n}{m} \equiv a_{1}}(\bmod N) \\
m^{k-1} \operatorname{sign}(m) \zeta_{N}^{a_{2} m} n>0
\end{gathered}
$$

If $k=2$ and $N>2$, the Fourier expansion of $G_{N, k}^{-a_{2} / a_{1}}(\tau)$ is given as follows (where $\alpha_{n}$ is defined similarly:

$$
G_{N, 2}^{-a_{2} / a_{1}}(\tau)=-\frac{2 \pi i}{N^{2}(\tau-\bar{\tau})}+\delta\left(\frac{a_{1}}{N}\right) \sum_{m_{2} \equiv a_{2}(N)}^{\prime} m_{2}^{-2}+\sum_{n>0} \alpha_{n}(N, 2, a) \exp (2 \pi i \tau n / N)
$$

Note that $G_{N, 2}^{-a_{2} / a_{1}}(\tau)$ transforms like a modular form although it is not holomorphic

Given this Fourier expansion, we can construct a basis for $\mathcal{E}_{k}(\Gamma(N))$ comprised of linear combinations of $G_{N, k}^{-a_{2} / a_{1}}$ such that for each $\Gamma(N)$-inequivalent cusp, exactly one element of the basis is nonvanishing. Using this above spanning set for $\widetilde{\mathcal{E}}_{2}(\Gamma(N))$ we give a basis for $\widetilde{\mathcal{E}}_{2}\left(\Gamma_{0}(N)\right)$. In doing so, we will follow the construction for Eisenstein series for arbitrary congruence subgroups given in $[19]$.

The dimension of this space $\operatorname{dim}\left(\mathcal{E}_{2}\left(\Gamma_{0}(N)\right)\right.$ is $\sigma_{\infty}-1$ where $\sigma_{\infty}(N)$ is the number of $\Gamma_{0}(N)$ inequivalent cusps [10]. Let

$$
\widetilde{\mathcal{E}}_{2}\left(\Gamma_{0}(N)\right)=\mathbb{C} E_{2} \oplus \mathcal{E}_{2}\left(\Gamma_{0}(N)\right)
$$

so that

$$
\operatorname{dim}\left(\widetilde{\mathcal{E}}_{2}\left(\Gamma_{0}(N)\right)=\sigma_{\infty}(N)\right.
$$


Definition 2.20. Suppose that $\left[\Gamma_{0}(N): \Gamma(N)\right]=d$ and denote by $A_{1}, \ldots A_{d}$ a set of coset representatives of $\Gamma_{0}(N)$ in $\Gamma(N)$. Then define a set of Eisenstein series for $\Gamma_{0}(N)$ by averaging over these coset representatives as follows:

$$
H_{\Gamma_{0}(N), k}^{-a_{2} / a_{1}}=\left.\sum_{n=1}^{d} H_{N, k}^{-a_{2} / a_{1}}\right|_{k} A_{n} .
$$

Lemma 2.21. The Eisenstein series $H_{\Gamma_{0}(N), 2}^{-a_{2} / a_{1}}$ is nonvanishing at all cusps $\Gamma_{0}(N)$-equivalent to $-a_{2} / a_{1}$ and vanishes at all other cusps.

Proof. The functions $H_{\Gamma_{0}(N), 2}^{-a_{2} / a_{1}}$ are given explicitly in $\S 2$ in 19 as an average of functions $G_{N, 2}^{-a_{2} / a_{1}}$ over pairs $\left(a_{1}, a_{2}\right)$. Following the Fourier expansions given in $\S 2.2$ in 19 , the series $G_{N, 2}^{-a_{2} / a_{1}}(\tau)$ is identical to $G_{N, k}^{-a_{2} / a_{1}}(\tau)$ for $k>2$ except for a non-holomorphic contribution $\frac{-2 \pi i}{N^{2}(\tau-\bar{\tau})}$. Because this component is independent of $a=\left(a_{1}, a_{2}\right)$, its contribution to the averaged function $H_{\Gamma_{0}(N), k}^{-a_{2} / a_{1}}$ from Equation (2.6) is also independent of $a$. The proof then follows mutatis mutandis as in Theorem 4, Ch. 7 of [19].

Lemma 2.22. If $a=\left(a_{1}, a_{2}\right)=(0,-1)$ corresponds to the cusp $\infty$, denoted by $H_{\Gamma_{0}(N), 2}^{\infty}$, then set $\left\{H_{\Gamma_{0}(N), 2}^{-a_{2} / a_{1}}\right\}$ as $a=\left(a_{1}, a_{2}\right)$ ranges over all cusps of $\Gamma_{0}(N)$ forms a basis for $\widetilde{\mathcal{E}}_{2}\left(\Gamma_{0}(N)\right)$.

Proof. Since $\operatorname{dim}\left(\widetilde{\mathcal{E}}_{2}\left(\Gamma_{0}(N)\right)\right)=\sigma_{\infty}(N)$, the set $\left\{H_{\Gamma_{0}(N), 2}^{-a_{2} / a_{1}}\right\}$ forms a basis for $\widetilde{\mathcal{E}}_{2}\left(\Gamma_{0}(N)\right)$ as desired.

Using this construction of $H_{\Gamma_{0}(N), k}^{-a_{2} / a_{1}}$, we can construct a normalized basis for $\widetilde{\mathcal{E}}_{2}\left(\Gamma_{0}(N)\right)$ :

Proposition 2.23. There is a set of weight 2 quasimodular Eisenstein forms for $\Gamma_{0}(N)$, say $F_{N, 2}^{-a_{2} / a_{1}}$, such that if $\operatorname{gcd}\left(a_{1}, a_{2}\right)=1$, then $F_{N, 2}^{-a_{2} / a_{1}}$ is 1 at the cusps $\Gamma_{0}(N)$-equivalent to the rational $-a_{2} / a_{1}$ and 0 at the other cusps. The set of linearly independent $F_{N, 2}^{-a_{2} / a_{1}}$ forms a basis for $\widetilde{\mathcal{E}}_{2}\left(\Gamma_{0}(N)\right)$.

Using these Eisenstein series, we can give an alternate representation of the holomorphic projection described in the previous section using Propstion 2.16. This occurs because $\widehat{\pi}_{h o l}\left(f_{E} \cdot \widehat{\mathfrak{Z}}_{E}\right)$ lies in the space of weight 2 quasimodular forms for $\Gamma_{0}(N)$ as per Proposition 2.15, and $f_{E} \cdot \widehat{\mathfrak{Z}}_{E}$ has moderate growth at all cusps (which will be shown later in Lemma 3.3 .

Corollary 2.24. Consider a strong Weil curve $E$ with conductor $N$, associated modular form $f_{E}$, and $\widehat{\mathfrak{Z}}_{E}$ as defined in (1.4). Then there are numbers $\alpha, \beta_{1}, \ldots, \beta_{\sigma_{\infty}(N)} \in \mathbb{C}$ such that

$$
\widehat{\pi}_{h o l}\left(f_{E} \cdot \widehat{\mathfrak{Z}}_{E}\right)=\alpha f_{E}+\sum_{i} \beta_{i} F_{N, 2}^{\rho(i)},
$$

$F_{N, 2}^{\rho(i)}$ is the weight 2 quasimodular form for $\Gamma_{0}(N)$ that takes the value 1 at the cusp $\rho(i)$ and vanishes at all other inequivalent cusps. 


\section{ProOfS OF THEOREMS}

Throughout this section, let $E$ be a strong Weil elliptic curve with conductor $N_{E}$ and associated weight 2 newform $f_{E}=\sum_{n=1}^{\infty} a_{E}(n) q^{n}$ of level $N=N_{E}$, where genus $\left(X_{0}(N)\right)=$ 1.

Definition 3.1. Consider $E$ as defined above. For each $q \mid N_{E}, q \in \mathbb{Z}^{+}$, we define the Atkin-Lehner involution $W_{q}$ to be

$$
W_{q}=\left[\begin{array}{cc}
q^{\alpha} a & b \\
N_{E} c & q^{\alpha} d
\end{array}\right]
$$

where $q^{\alpha} \| N_{E}$ and $a, b, c, d \in \mathbb{Z}$ such that $W_{q}$ has determinant $q^{\alpha}$.

We can use these $W_{q}$ to understand the Fourier expansion of $\widehat{\mathfrak{Z}}_{E}$ and ultimately the holomorphic projection $\widehat{\pi}_{\text {hol }}\left(f_{E} \cdot \widehat{\mathfrak{Z}}_{E}\right)$ at the $\Gamma_{0}(N)$ inequivalent cusps.

Proposition $3.2(\S 1[1])$. Consider $E, f_{E}$ as above. For all $W_{q}$, there exists $\lambda_{q} \in\{ \pm 1\}$, the Atkin-Lehner eigenvalue of $f_{E}$ such that $\left.f_{E}\right|_{2} W_{q}=\lambda_{q} f_{E}$.

Given this characterization of the Atkin-Lehner involution, we can understand the Fourier expansion of $\widehat{\mathfrak{Z}}_{E}$ at the cusps:

Theorem 3.3 (§1 [1]). Consider $E, f_{E}$ as above. If $N$ is squarefree and $q \mid N_{E}$, then

$$
\begin{gathered}
\left.\widehat{\mathfrak{Z}}_{E}\right|_{0} W_{q}=\widehat{\mathfrak{Z}}_{E}^{+}\left(\lambda_{q}\left(\mathcal{E}_{E}(z)-\Omega_{q}\left(f_{E}\right)\right)\right)-\frac{1}{4 \pi|| f_{E} \|^{2}} \cdot \overline{\lambda_{q}\left(\mathcal{E}_{E}(z)-\Omega_{q}\left(f_{E}\right)\right)} \\
\Omega_{q}\left(f_{E}\right)=-2 \pi i \int_{W_{q}^{-1} \infty}^{\infty} f_{E}(z) d z
\end{gathered}
$$

Consider some cusp representative $\rho(i)$ of $\Gamma_{0}(N)$. Then, define $\sigma_{i}$ (with associated $\lambda_{i}, \Omega_{i}$ ) to be the Atkin-Lehner involution such that $\sigma_{i} \rho(i)=\infty$. Using this, we now prove the following lemmas that will enable us to show Theorem 1.1 .

Lemma 3.4. Consider $E$ as defined above. The weak harmonic Maaß form $\widehat{\mathfrak{Z}}_{E}(z)$ is good for $f_{E}(z)$, where $f_{E}(z)$ is the weight 2 newform associated to $E$.

Proof. Note that as per Lemma 2.9. $\widehat{\mathfrak{Z}}_{E}^{+}$has no poles on the upper half plane and consequently, $M_{E}(z)=0$ in the case we consider (where genus $\left(X_{0}(N)\right)=1$ ). If $E$ has CM, then $\widehat{\mathfrak{Z}}_{E}(z)$ is good for $f_{E}$ by Theorem 6 of $[9$. If not, $N$ is squarefree. Note that by construction of $\widehat{\mathfrak{Z}}_{E}(z)$, this harmonic Maaß form satisfies condition (3) to be good.

For squarefree conductor $N$, the Weierstrass mock module form has a pole arising from the $\frac{1}{z}$ term in the Laurent expansion of the evaluation of the Weierstrass $\zeta$ function at $\mathcal{E}_{f_{E}}(z)$. This has no constant term, and thus $\widehat{\mathfrak{Z}}_{E}^{+}(z)=q^{-1}+O(1)$.

Following the proof in [1] of Theorem 3.3 .

$$
\Omega_{q}\left(f_{E}\right)=\mathcal{E}_{E}(z)-\lambda_{q} \mathcal{E}_{E}\left(W_{q} z\right), \quad z \in \mathcal{H}
$$

Therefore, by choosing $z \in \mathcal{H}$ so that $\Im(z), \Im\left(W_{q} z\right)$ are comparable (to give tight approximations), we can evaluate the Eichler integrals to verify $\Omega_{q}\left(f_{E}\right)$ is nonvanishing for each of the finitely many desired cases $\left(N_{E}=11,14,15,17,19,21\right)$. 
For example, in the case of conductor $N_{E}=14$,

$$
W_{14}=\left[\begin{array}{cc}
0 & -1 \\
14 & 0
\end{array}\right], \quad z=\frac{1}{\sqrt{14}} i
$$

gives $\Omega_{14}=0.3302$,

$$
W_{7}=\left[\begin{array}{cc}
7 & 1 \\
42 & 7
\end{array}\right], \quad z=0.1032 i
$$

gives $\Omega_{7}=0.6862$, and

$$
W_{2}=\left[\begin{array}{cc}
4 & 1 \\
14 & 4
\end{array}\right], \quad z=0.1091 i
$$

gives $\Omega_{2}=-1.3255$.

Following Theorem 3.3, $\left.\widehat{\mathfrak{Z}}_{E}\right|_{0} W_{q}$ has mock modular form contribution $\widehat{\mathfrak{Z}}_{E}^{+}\left(\lambda_{q}\left(\mathcal{E}_{E}(z)-\right.\right.$ $\left.\left.\Omega_{q}\left(f_{E}\right)\right)\right)$. Since $\Omega_{q}\left(f_{E}\right)$ is never zero for $q \mid N, \widehat{\mathfrak{Z}}_{E}(z)=c+O(q)$, for some constant $c$ as desired, and thus $\widehat{\mathfrak{Z}}_{E}(z)$ is good for $f_{E}$.

Lemma 3.5. Consider $E$ as defined above. Then $\widehat{\mathfrak{Z}}_{E}^{+}$vanishes at all cusps not equivalent to the cusp $\infty$ of $\Gamma_{0}(N)$.

Proof. If $E$ satisfies the above conditions, then $\widehat{\mathfrak{Z}}_{E}$ is good for $f_{E}$. In particular, $\widehat{\mathfrak{Z}}_{E}$ has a pole at $\infty$ and constant principal part at the cusps of $\Gamma_{0}(N)$ by Lemma 3.4. On the other hand, the index -1 Maaß-Poincaré series $Q(-1,2, N ; z)$ has a pole at $\infty$ and zero principal part at the cusps (Proposition 2.12). The difference $\widehat{\mathfrak{Z}}_{E}(z)-Q(-1,2, N ; z$ ) is a weight 0 harmonic Maass form with no poles and constant value at each of the cusps. The differential operator $\xi_{0}$ maps harmonic Maaß forms of weight $2-k$ to cusp forms of weight $k$. Then since the dimension of $S_{2}\left(\Gamma_{0}(N)\right)$ is 1 , we have

$$
\xi_{0}\left(\widehat{\mathfrak{Z}}_{E}(z)-Q(-1,2, N ; z)\right)=c \cdot f_{E}(z)
$$

for some constant $c$. However, by Lemma 2.5 , since $\widehat{\mathfrak{Z}}_{E}(z)-Q(-1,2, N ; z)$ has constant principal part at all cusps, we find that $c=0$. This implies that the difference is holomorphic, and so is constant. Then $\widehat{\mathfrak{Z}}_{E}(z)$ and $Q(-1,2, N ; z)$ are equal up to an additive constant since both have leading term $q^{-1}$, as are their holomorphic parts $\widehat{\mathfrak{Z}}_{E}^{+}(z)$ and $Q^{+}(-1,2, N ; z)$. Thus, as in 18, since $Q^{+}(-1,2, N ; z)$ vanishes at all cusps not equivalent to infinity by Lemma $2.12, \widehat{\mathfrak{Z}}_{E}^{+}(z)$ vanishes at all cusps not equivalent to infinity.

Using this Lemma, we are able to prove the first theorem.

Proof of Theorem 1.1. From Lemma 2.17 and Lemma 2.24, we obtain that

$$
\widehat{\pi}_{\text {hol }}\left(\widehat{\mathfrak{Z}}_{E} \cdot f_{E}\right)(z)=f_{E}(z) \cdot \widehat{\mathfrak{Z}}_{E}^{+}(z)-\frac{\pi}{\operatorname{vol}\left(\Lambda_{E}\right)} \sum_{h=1}^{\infty} D_{f_{E}}(h ; 1) q^{h}=\alpha f_{E}+\sum_{i} \beta_{i} F_{N, 2}^{\rho(i)}(z) \text {. }
$$

We can compute the holomorphic projection at each cusp $\rho(i)$ using Atkin-Lehner involutions as in Theorem 3.3 (since $N$ here is squarefree), which gives the values $\beta_{i}$. Note that the $L$-series generating function $\mathbb{L}_{f_{E}}(z)$ vanishes at the cusp $\infty$ and $f_{E}$ vanishes at all cusps $\rho(i)$. Applying Lemma 3.5. $\widehat{\mathfrak{Z}}_{E}^{+}$vanishes at all cusps inequivalent to the cusp $\infty$ of $\Gamma_{0}(N)$. Consequently the holomorphic projection vanishes at all cusps not $\Gamma_{0}(N)$-equivalent to $\infty$ 
and is 1 at the $\operatorname{cusp} \infty$. If we let $\rho(1)$ denote the $\operatorname{cusp} \infty, \beta_{i}=0$ for $i \neq 1$ and $\beta_{1}=1$. Using this, we can compute $\alpha$ by equating the first Fourier coefficient of both expressions for $\widehat{\pi}_{\text {hol }}$. Rearranging gives the desired expression for $\mathbb{L}_{f_{E}}(z)$.

We also consider the case where $E$ as defined in the beginning has complex multiplication, which will give an analogous result for the shifted convolution $L$-series values.

Lemma 3.6. Consider $E$ as defined above which also has complex multiplication and thus conductor $N \neq 49$ with associated modular form $f_{E}(z)$. Then $D_{f_{E}}(h ; 1)=0$ if $h \not \equiv 0$ $\left(\bmod n_{0}\right)$.

Proof. Suppose that $E$ has complex multiplication. Then if $p$ is a prime inert in the CM field, $a_{E}(p)=0$. Since $f_{E}$ is a weight 2 newform, its coefficients are multiplicative. As in $\S 5$ of [7], $a_{E}(n)=0$ for all $n \not \equiv 1\left(\bmod n_{0}\right)$ where $n_{0} \mid N$ is a curve-dependent value always at least 3. For example, in the case of the $\Gamma_{0}(27)$-optimal elliptic curve with complex multiplication, $n_{0}=3$. Now recall that $D_{f_{E}}(h ; 1)$ is defined by

$$
D_{f_{E}}(h ; 1)=\sum_{n=1}^{\infty} a_{E}(n+h) a_{E}(n)\left(\frac{1}{n+h}-\frac{1}{n}\right) .
$$

Suppose that $D_{f_{E}}(h ; 1)$ is nonvanishing. Then both $a_{E}(n+h)$ and $a_{E}(n)$ must be nonvanishing and thus $n+h, n \equiv 1\left(\bmod n_{0}\right)$. This yields $h \equiv 0\left(\bmod n_{0}\right)$.

Remark. For $N=49$, the support of the Hecke eigenvalues $a_{E}(n)$ are at $n \equiv 1,2,4(\bmod 7)$. In this case, the proof does not hold, and the $L$-series has support everywhere. Thus, although the theorem appears to hold numerically in the $N=49$ case, this approach does not yield the desired result.

Lemma 3.7. Consider $E$ with complex multiplication as defined at the beginning of the section with conductor $N \neq 49$ and associated modular form $f_{E}$. Then there exists some $n_{0} \geq 3$ with $n_{0} \mid N$ such that $\widehat{\pi}_{h o l}\left(f_{E} \cdot \widehat{\mathfrak{Z}}_{E}\right)[h]=0$ if $h \not \equiv 0\left(\bmod n_{0}\right)$.

Proof. The derivative of the Weierstrass mock modular form $\widehat{\mathfrak{Z}}_{E}^{+}$for the three strong Weil curves of conductor $N=27,32$, and 36 are given as eta-quotients in the following table (see [9]).

\begin{tabular}{|c|c|c|}
\hline$N$ & $\widehat{\mathfrak{Z}}_{E}^{+}(\cdot)$ & $q \frac{d}{d q}\left(\widehat{\mathfrak{Z}}_{E}^{+}\right)$ \\
\hline 27 & $q^{-1}+\frac{1}{2} q^{2}+\frac{1}{5} q^{5}+\frac{3}{4} q^{8}+\cdots$ & $-\frac{\eta(3 \tau) \eta(9 \tau)^{6}}{\eta(27 \tau)^{3}}$ \\
\hline 32 & $q^{-1}+\frac{2}{3} q^{3}+\frac{1}{7} q^{7}-\frac{2}{11} q^{11}+\cdots$ & $-\frac{\eta^{2}(4 \tau) \eta^{6}(16 \tau)}{\eta^{4}(32 \tau)}$ \\
\hline 36 & $q^{-1}+\frac{3}{5} q^{5}+\frac{1}{11} q^{11}+\cdots$ & $-\frac{\eta^{3}(6 \tau) \eta(12 \tau) \eta(18 \tau)}{\eta^{3}(36 \tau)}$ \\
\hline
\end{tabular}

The support of the derivative $q \frac{d}{d q}\left(\widehat{\mathfrak{Z}}_{E}^{+}\right)$is the same as the support of the Weierstrass mock modular form. The form of the eta-quotients indicates that the support for $\widehat{\mathfrak{Z}}_{E}^{+}$for $N=27$ 
is $-1(\bmod 3)$, for $N=32$ is $-1(\bmod 4)$, and for $N=36$ is $-1(\bmod 6)$. Let $n_{0}=3,4,6$ for $N=27,32,36$ respectively.

Each of these curves has complex multiplication, and their associated modular forms $f_{E}$ have support (are nonvanishing) only at $1\left(\bmod n_{0}\right)$. Thus the product of the Weierstrass mock modular form $\widehat{\mathfrak{Z}}_{E}^{+}$and $f_{E}$ has support at $0\left(\bmod n_{0}\right)$.

By Lemma 3.6, the $L$-series is also only supported at $0\left(\bmod n_{0}\right)$, which yields that the holomorphic projection

$$
\pi_{\mathrm{hol}}\left(f_{E} \cdot \widehat{\mathfrak{Z}}_{E}^{+}\right)=f_{E} \cdot \widehat{\mathfrak{Z}}_{E}^{+}-\frac{\pi}{\operatorname{vol}\left(\Lambda_{E}\right)} \mathbb{L}_{f_{E}}(z)
$$

has support only at $0\left(\bmod n_{0}\right)$.

Now, we can prove Theorem 1.2 .

Proof of Theorem 1.2. Following the proof for Theorem 1.1, we obtain that $\beta_{1}=1$, and $\beta_{i}=0$ for $i \neq 1$ in Equation (2.7). Since $f_{E}$ is only supported at coefficients $1 \bmod n_{0}$, where the left hand side is not supported (see Lemma 3.7), $\alpha=0$. Thus, we have that

$$
\widehat{\pi}_{\text {hol }}\left(\widehat{\mathfrak{Z}}_{E} \cdot f_{E}\right)(z)=F_{N, 2}^{\infty}(z) \text {. }
$$

We can compute the desired closed form expression for the shifted-convolution $L$-series values:

$$
\mathbb{L}_{f_{E}}(z)=\frac{\operatorname{vol}\left(\Lambda_{E}\right)}{\pi}\left(\left(f_{E}(z) \cdot \widehat{\mathfrak{Z}}_{E}^{+}(z)\right)-F_{N, 2}^{\infty}(z)\right)
$$

Remark. Note that results such as the above theorems may be able to be transformed to give recurrence relations on the modular form coefficients, giving expressions for the Fourier coefficients analogous to those obtained in obtained in $\S 12$ of [16] and 8].

\section{EXAmples}

4.1. Conductor $N=11$. Consider the modular curve $X_{0}(11)$ of dimension 1 . There is a single isogeny class of elliptic curves, and the strong Weil curve is given by the Weierstrass equation

$$
E: y^{2}+y=x^{3}-x^{2}-10 x-20 \text {. }
$$

Numerically, we find that $S\left(\Lambda_{E}\right)=0.38124 \ldots$ Using this and the Fourier expansion of the Weierstrass $\zeta$-function, the corresponding weight 0 mock modular form $\widehat{\mathfrak{Z}}_{E}^{+}(z)$ is given by

$$
q^{-1}+1+0.9520 \ldots q+1.547 \ldots q^{2}+0.3493 \ldots q^{3}+1.976 \ldots q^{4}-2.609 \ldots q^{5}+O\left(q^{6}\right) .
$$

Using the formula given in Proposition 2.17, one can compute the $L$-series numerically. Using 100000 coefficients of $f_{E}$, we have the Fourier expansion $\mathbb{L}_{f_{E}}(z)=\sum_{n=1}^{\infty} D_{f_{E}}(h ; 1) q^{h}$ :

$$
\mathbb{L}_{f_{E}}(z)=0.7063 \ldots q+1.562 \ldots q^{2}+0.0944 \ldots q^{3}+1.237 \ldots q^{4}-2.026 \ldots q^{5}+O\left(q^{6}\right)
$$

The space of weight 2 quasimodular Eisenstein series for $\Gamma_{0}(11)$ is 2 dimensional, and a basis is given by $F_{11,2}^{0}$ and $F_{11,2}^{\infty}$, where $F_{11,2}^{\infty}$ is

$$
F_{11,2}^{\infty}=1+\frac{1}{5} q+\frac{3}{5} q^{2}+\frac{4}{5} q^{3}+\frac{7}{5} q^{4}+\frac{6}{5} q^{5}+\frac{12}{5} q^{6}+O\left(q^{7}\right) .
$$


Then, if we take $\alpha=.0016, \beta_{1}=1, \beta_{2}=0$, we obtain another way to retreive the $L$-series values in Equation 4.1):

$$
\begin{aligned}
& \quad \frac{\operatorname{vol}\left(\Lambda_{E}\right)}{\pi}\left(\left(f_{E} \cdot \widehat{\mathfrak{Z}}_{E}^{+}\right)-\alpha f_{E}-F_{11,2}^{\infty}\right)= \\
& 0.706 \ldots q+1.562 \ldots q^{2}+0.0930 \ldots q^{3}+1.234 \ldots q^{4}-2.024 \ldots q^{5}+O\left(q^{6}\right) .
\end{aligned}
$$

Note that to the accuracy of the computations, the value $\alpha \approx 0$ in this case.

4.2. Conductor $N=27$. Recall the strong Weil curve of conductor 27 given by the Weierstrass equation $E_{27}: y^{2}+y=x^{3}-7$ (Cremona label 27a1). The weight 2 modular form associated with $E_{27}$ is given by

$$
f_{E_{27}}=q-2 q^{4}-q^{7}+5 q^{13}+4 q^{16}-7 q^{19}+O\left(q^{20}\right) .
$$

Using the Fourier expansion of the Weierstrass $\zeta$-function, the weight 0 mock modular form associated to $\widehat{\mathfrak{Z}}_{E_{27}}^{+}(z)$ is given by

$$
\widehat{\mathfrak{Z}}_{E_{27}}^{+}(z)=q^{-1}+\frac{1}{2} q^{2}+\frac{1}{5} q^{5}+\frac{3}{4} q^{8}-\frac{6}{11} q^{11}-\frac{1}{2} q^{14}+O\left(q^{17}\right) .
$$

The holomorphic projection is given by

$$
\widehat{\pi}_{\text {hol }}\left(f_{E} \cdot \widehat{\mathfrak{Z}}_{E_{27}}^{+}\right)(z)=1+3 q^{9}+9 q^{18}-12 q^{27} \ldots
$$

On the other hand, we find that the normalized element of the weight 2 quasimodular forms for $\Gamma_{0}(27)$ that vanishes at all cusps but $\infty$ is given by

$$
F_{27,2}^{\infty}=1+3 q^{9}+9 q^{18}-12 q^{27} \ldots
$$

This agrees with the computation for the holomorphic projection. Then the generating function $\mathbb{L}_{f}(z)$ can also be computed by Theorem 1.2 , giving arbitrary precision computations for these slowly convergent shifted convolution $L$-series values that could only previously be computed term-by-term.

\section{ACKNOWLEDGEMENTS}

The authors would like to thank the NSF (grant DMS-1250467) and the Emory REU (especially Dr. Mertens and Professor Ono) for their support. We would also like to thank the anonymous referee for their careful review and helpful suggestions.

\section{REFERENCES}

[1] C. Alfes, M. Griffin, K. Ono, and L. Rolen. Weierstrass mock modular forms and elliptic curves. Research in Number Theory, 1(1):1-31, 2015.

[2] O. Beckwith. Asymptotic bounds for special values of shifted convolution dirichlet series. To appear, Proceedings of the American Mathematical Society, 2016.

[3] C. Breuil, B. Conrad, F. Diamond, and R. Taylor. On the modularity of elliptic curves over $\mathbb{Q}$ : wild 3-adic exercises. Journal of the American Mathematical Society, 14(4):843-939, 2001.

[4] K. Bringmann, M. H. Mertens, and K. Ono. p-adic properties of modular shifted convolution Dirichlet series. Proceedings of the American Mathematical Society, 144(4):1439-1451, 2016.

[5] K. Bringmann and K. Ono. Coefficients of harmonic Maass forms. In Partitions, q-Series, and Modular Forms, pages 23-38. Springer, 2012. 
[6] J. H. Bruinier and J. Funke. On two geometric theta lifts. Duke Mathematical Journal, 125(1):45-90, 2004.

[7] J. H. Bruinier, K. Ono, and R. C. Rhoades. Differential operators for harmonic weak Maass forms and the vanishing of Hecke eigenvalues. Mathematische Annalen, 342(3):673-693, 2008.

[8] D. Choi. Poincaré series and the divisors of modular forms. Proceedings of the American Mathematical Society, 138(10):3393-3403, 2010.

[9] A. Clemm. Modular forms and Weierstrass mock modular forms. Mathematics, 4(1):5, 2016.

[10] F. Diamond and J. Shurman. A first course in modular forms, volume 228. Springer Science \& Business Media, 2006.

[11] M. Eichler. Eine Verallgemeinerung der Abelschen Integrale. Mathematische Zeitschrift, 67(1):267-298, 1957.

[12] B. H. Gross and D. B. Zagier. Heegner points and derivatives of $L$-series. Inventiones mathematicae, 84(2):225-320, 1986.

[13] J. Hoffstein, T. A. Hulse, and A. Reznikov. Multiple Dirichlet series and shifted convolutions. Journal of Number Theory 161:457-533, 2016.

[14] P. Guerzhoy. A mixed mock modular solution of the Kaneko-Zagier equation. The Ramanujan Journal, 36(1-2):149-164, 2015.

[15] M. H. Mertens and K. Ono. Special values of shifted convolution Dirichlet series. Mathematika, 62(1):47$66,2016$.

[16] K. Ono. Unearthing the visions of a master: harmonic Maass forms and number theory. Curr. Dev. Math., 2008:347-454, 2009.

[17] R.A. Rankin. Contributions to the theory of Ramanujan's function $\tau(n)$ and similar arithmetical functions. Math. Proc. Camb. Phil. Soc., 35-03:357-372, 1939.

[18] R. C. Rhoades. Linear relations among Poincaré series via harmonic weak Maaßforms. The Ramanujan Journal, 29(1-3):311-320, 2012.

[19] B. Schoeneberg. Elliptic modular functions: an introduction, volume 203. Springer Science \& Business Media, 2012.

[20] A. Selberg, V. Bjerknes, J. Molland. Bemerkungen über eine Dirichletsche Reihe, die mit der Theorie der Modulformen nahe verbunden ist. Cammermeyer i Komm., 1940.

[21] P. G. Shimura. Sur les intégrales attachées aux formes automorphes. Journal of the Mathematical Society of Japan, 11(4):291-311, 1959.

[22] J. Sturm. Projections of $\mathcal{C}^{\infty}$ automorphic forms. Bulletin of the American Mathematical Society, $2(3): 435-439,1980$.

[23] E. T. Whittaker. An expression of certain known functions as generalized hypergeometric functions. Bulletin of the American Mathematical Society, 10(3):125-134, 1903.

[24] D. Zagier. Modular parametrizations of elliptic curves. Canad. Math. Bull, 28(3):372-384, 1985.

[25] S. Zwegers. Mock theta functions. PhD Thesis, Universiteit Utrecht 2002.

Massachusetts Institute of Technology, Department of Mathematics, Cambridge, MA

E-mail address: asra@mit.edu

Stanford University, Department of Mathematics, Stanford, CA 94305

E-mail address: nityam@stanford.edu 